\title{
Jean Norton Cru, littérature et témoignages de la Première Guerre mondiale
}

Jean Norton Cru, Literatur und Zeugnisse zum Ersten Weltkrieg

Jean Norton Cru, literature and testimonies of the First World War

Jean-Marie Guillon

\section{(2)enEdition}

\section{Journals}

Édition électronique

URL : http://journals.openedition.org/ceg/2077

DOI : $10.4000 /$ ceg. 2077

ISSN : 2605-8359

\section{Éditeur}

Presses Universitaires de Provence

\section{Édition imprimée}

Date de publication : 15 juin 2014

Pagination : 187-196

ISSN : 0751-4239

Référence électronique

Jean-Marie Guillon, « Jean Norton Cru, littérature et témoignages de la Première Guerre mondiale », Cahiers d'Études Germaniques [En ligne], 66 | 2014, mis en ligne le 17 décembre 2017, consulté le 19 novembre 2020. URL : http://journals.openedition.org/ceg/2077 ; DOI : https://doi.org/10.4000/ceg 2077 


\title{
Jean Norton Cru, littérature et témoignages de la Première Guerre mondiale
}

\author{
Jean-Marie GUILLON
}

Université d'Aix-Marseille

Cette contribution entend attirer l'attention sur un acteur majeur de la représentation de la Grande Guerre et du combattant, Jean Norton Cru, dont l'œuvre a fait couler beaucoup d'encre depuis la fin des années vingt. Elle reste au centre de polémiques contemporaines qui touchent tant aux témoignages de guerre qu'à l'utilisation de la littérature comme source historique ou aux représentations de la guerre.

Ces polémiques ont été suscitées par la publication en 1929 d'un fort ouvrage, intitulé Témoins ${ }^{1}$, dans lequel Cru dissèque de façon très pointilleuse, mais aussi avec une méthode systématique et novatrice (même si elle est contestable), la littérature française publiée depuis la guerre et dont la guerre est l'objet, soit au total trois cents récits de nature diverse. Cet ouvrage, publié chez un petit éditeur, a un retentissement tel que Gallimard édite l'année suivante dans la collection de la nrf un texte du même auteur aux dimensions plus modestes, intitulé $D u$ témoignage, où sont rassemblées principalement les considérations de méthode qu'il préconise en matière de sélection du "bon" témoignage ${ }^{2}$. Ce sont ces ouvrages qui provoquent les débats ${ }^{3}$. Mais Témoins est devenu, sinon une référence, du moins un jalon qui a marqué l'historiographie de la Grande Guerre et l'historiographie tout court, surtout à partir du moment où l'on a accordé à la mémoire, à ses

\footnotetext{
1 Jean Norton CRU, Témoins. Essai d'analyse et de critique des souvenirs des combattants édités en français de 1915 à 1928, Paris, Les Étincelles, 1929, rééd. Nancy, Presses universitaires de Nancy, 2006.

2 Jean Norton CRU, Du témoignage, Paris, Gallimard, nrf, 1930. Diverses rééditions par la suite, notamment la réédition partielle complétée par l'article biographique sur « Jean Norton Cru » par sa sœur Hélène VOGEL, Paris, Jean-Jacques Pauvert (coll. « Libertés »), 1966.

${ }_{3}^{3}$ Sur cette réception, Frédéric Rousseau, Le procès des témoins de la Grande Guerre. L'affaire Norton Cru, Paris, Seuil, 2003. Nous ne reviendrons pas sur la polémique qui a opposé au sujet de l'interprétation de son œuvre et de son apport les historiens de la Première Guerre mondiale.
} 
représentations et à leur évolution dans le temps l'attention que l'on sait. Jean Norton Cru a joué « un rôle pionnier » en voulant « donner aux témoignages le statut de documents historiques ${ }^{4} »$. Ce faisant, il a voulu aussi donner la parole à la piétaille qui fait la guerre et la subit, et qui, de son point de vue, n'avait pas accès à l'Histoire. Dans l'engouement suscité par l'histoire " orale » il y a trente ans, ce chantre du témoignage et d'une autre histoire, celle d'en bas contre l'histoire "officielle », celle d'en haut, est même apparu comme une sorte de précurseur.

Précisons que si l'œuvre de Cru était bien connue, le personnage lui-même l'était beaucoup moins. Sa sœur, Hélène Vogel, professeur de français au lycée Montgrand à Marseille et amie de Pierre Guiral qui enseignait l'histoire contemporaine à la Faculté des Lettres d'Aix-en-Provence, avait écrit un article biographique publié par les Annales de la Faculté des Lettres et Sciences humaines d'Aix-en-Provence en $1961^{5}$, qui constituait avec les quelques notations qu'il avait faites lui-même dans Témoins sur son parcours de soldat le seul éclairage disponible. La découverte que notre équipe a réalisée de la correspondance de guerre de Jean Norton Cru et l'enquête conduite par Marie-Françoise Attard-Maraninchi permettent enfin d'éclairer l'homme, son milieu familial et son parcours ${ }^{6}$.

\section{Qui est Jean Norton Cru?}

Il est né en 1879 et a donc 35 ans en 1914 : c'est pourquoi il est mobilisé dans la territoriale, puis l'infanterie de réserve. Mais il est sur le front d'octobre 1914 jusqu'en janvier 1917 et s'est retrouvé à plusieurs reprises dans les premières lignes. Il est passé par le secteur de Saint-Mihiel dans la Meuse, puis par l'Argonne, la Champagne, Verdun à deux reprises, le Chemin des Dames, et, même quand il est affecté comme interprète auprès de l'armée américaine, il participe à l'entrée au combat de son régiment en Lorraine.

Lorsqu'il est mobilisé, il se trouve aux États-Unis, dans le Massachussetts où il enseigne depuis 1908. Il est professeur de littérature française à Williams College, une institution notoire, destinée à la formation de l'élite WASP située à Williamstown. Ses trois autres frères enseignent également aux États-Unis : l'un, Paul, au Hunter College de New York, et les deux autres, Albert et Loyalty, à Columbia, toujours à New York. Ces deux derniers sont mobilisés comme lui pour participer à la guerre en France. Jean

\footnotetext{
${ }^{4}$ Antoine Prost, Jay WINTER, Penser la Grande Guerre. Un essai d'historiographie, Paris, Seuil (coll. « Points Histoire »), 2004, p. 116.

Hélène VOGEL, « Jean Norton Cru. Sa vie par rapport à Témoins », Annales de la Faculté des Lettres et Sciences humaines d'Aix-en-Provence, 1961, t. XXXV, p. 37-68.

${ }^{6}$ Marie-Françoise ATTARD-MARANINCHI et Roland CATY (éds.), Jean Norton Cru, Lettres du front et d'Amérique 1914-1919, Aix-en-Provence, PUP, 2007, ouvrage dont j'ai rédigé l'introduction.
} 
Norton est l'aîné de la famille. Ses frères et sœurs, qui sont tous enseignants, ont suivi un parcours académique brillant puisque deux d'entre eux, Loyalty et Hélène, ont été admis à l'École normale supérieure et sont agrégés, lui d'anglais et elle de lettres. C'est Loyalty qui, après l'ENS, est arrivé le premier aux États-Unis et qui a fait venir ses frères à Williamstown. Il est vrai que la famille est bilingue. Si leur père est issu d'un milieu très modeste, leur mère anglaise vient d'une famille aisée et cultivée. C'est autour d'elle que le clan familial se rassemble après qu'elle a divorcé ${ }^{7}$. En effet, le père, bien que pasteur, apparait comme un individu fruste et aux mœurs dissolues, et il est rejeté ${ }^{8}$. La culture maternelle et l'expérience américaine donnent à Jean Norton Cru une certaine distance critique vis-à-vis de la société française. Il est à la fois dedans - et son patriotisme ne faiblit jamais - et dehors. Étant l'âné et compte tenu des tensions familiales, il n'a pas pu suivre le cursus d'excellence de ses frères. Titulaire du baccalauréat en 1899, il a passé le certificat d'aptitude à l'enseignement de l'anglais après son service militaire, puis il a continué ses études de lettres ${ }^{9}$ tout en enseignant aux États-Unis et un an en Algérie. De ce fait, on sent qu'il a une revanche à prendre par rapport au monde universitaire. C'est une évidence dans son œuvre, non qu'il nourrisse du ressentiment, mais il entend par la méthode qu'il met au point et par les résultats qu'il publie en remontrer, notamment aux tenants d'une histoire qu'il estime trop "officielle». Comme je l'ai écrit en introduisant sa correspondance de guerre, Témoins est la thèse qu'il n'a pu faire.

Le titre de cet ouvrage et les préoccupations qu'il reflète ne sont pas neutres. Il s'agit certes de donner la parole aux "vrais" combattants, à ses camarades de tranchée, de se faire leur porte-parole, mais la Parole est pour ce protestant fervent bien plus qu'un mot banal. Elle a un caractère sacré et porter cette parole est, pour lui, la mission - au sens le plus fort - qu'il s'est assignée $^{10}$. On ne peut comprendre le personnage - raisonneur, moraliste, puritain ne cessant de donner des leçons à ses sœurs, par exemple, mais en même temps libéral et ouvert sur bien des plans et notamment celui de l'égalité des sexes - si l'on ignore sa culture réformée. Elle vient de sa mère et marque profondément toute cette famille, où Jean Norton joue un peu le rôle de mentor. La correspondance de guerre conservée est celle qu'il adresse à ses sœurs. Elle illustre l'affection qu'il porte à Hélène, qui se fera la

\footnotetext{
${ }^{7}$ Pour plus de précisions sur la famille, je renvoie au travail de Marie-Françoise ATTARDMARANINCHI, Jean Norton Cru, Lettres du front, p. 19 sq. Paul et Albert feront, comme Jean Norton, toute leur carrière aux États-Unis. Jean Norton deviendra Assistant Professor en 1919 , puis Associate Professor en 1932, toujours à Williams College. Loyalty s'installera après guerre à Londres comme correspondant du grand journal diplomatique Le Temps et directeur de la Maison de l'Institut de France.

${ }^{8}$ Le père a exercé un ministère en Nouvelle-Calédonie de 1883 à 1891, puis a été contraint de revenir en métropole où, à l'évidence, son mode de vie reste dissolu.

9 Jusqu'au DES (diplôme d'études supérieures) soutenu à la Sorbonne en 1913, année où il est également admissible à l'agrégation d'anglais.

${ }^{10}$ Ce que Rémy CAZALS fait remarquer à juste titre (" Quelques pierres apportées au chantier », Annales du Midi, t. 112, n 232, octobre-décembre 2000, p. 443).
} 
gardienne de sa mémoire, et à Alice, qui enseigne aussi à Marseille, au lycée Longchamp, où elle est professeur d'anglais.

\section{Aux origines de Témoins}

Le projet de Témoins est né dans la tranchée et il est significatif de la prise de conscience par son auteur de la réalité de la guerre et des travestissements de la propagande ${ }^{11}$. Sa correspondance le prouve. Comme la plupart de ses camarades, Cru accepte la mobilisation et partage les illusions communes sur le combat à venir. Or, dans les tranchées, au fil des mois, il découvre que la guerre a un autre visage que celui que lui présentaient les œuvres littéraires dont il était nourri et qui l'ont induit en erreur. Mais elle ne ressemble pas non plus à ce qu'en disent les journaux ou les premiers ouvrages qui prétendent la décrire. Elle ne correspond pas aux communiqués d'état-major. "Jamais la guerre n'a été plus sauvage, plus barbare, plus inhumaine qu'aujourd'hui », écrit-il le 9 janvier $1915^{12}$. Les mensonges de la propagande, les «élucubrations » des journaux le révoltent. Ils heurtent ses convictions et il n'aura de cesse de les traquer. S'il condamne « les procédés inqualifiables de l'ennemi » (2 mars 1915), il dénonce les mythes qui donnent une fausse idée de lui : « La frousse est humaine et non allemande : chacun en a sa part et il est difficile qu'on soit Français ou Boche de voir sans crainte une charge à la baïonnette " (6 juillet 1915) ${ }^{13}$. Il pourfend déjà ce qu'il va appeler dans Témoins les "idées fausses sur la guerre ${ }^{14}$ » et, tout particulièrement, le dénigrement imbécile de l'ennemi, les "légendes héroïques", le combat au corps à corps, alors que la guerre devient anonyme et que la mort se donne à distance, ce qui rend les choses encore plus angoissantes. Il écrira dans Témoins que les auteurs qui font un grand usage de la baïonnette sont « tous ceux qui inventent leurs souvenirs ${ }^{15} »$.

Il n'est pas mu par une idéologie politique et se situe davantage sur un plan religieux ou moral. La politique n'a aucune place dans sa correspondance. Il récuse toute idéologie déraisonnable, pouvant donc conduire au fanatisme. Il rejette le nationalisme, forcément guerrier, le révolutionnarisme, comme les différentes versions du pacifisme. C'est la psychologie de l'individu qui l'intéresse, les réactions de ses camarades. La vérité de la guerre se situe pour lui à ce niveau-là. Il est par toutes ses fibres un protestant libéral et il se veut homme des Lumières. Ses engagements sont d'abord éthiques. Sa mission est de pourfendre les faux prophètes et les marchands du Temple et de proclamer ce qu'il est persuadé être le vrai. Faire

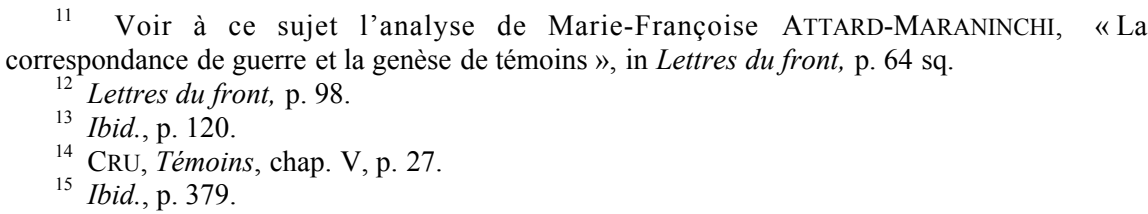


connaître la vérité sur la guerre permettra de dissiper la tromperie et donc de faire perdre ses attraits éventuels au combat. Ce pédagogue impénitent a confiance en l'homme. Il est par certains côtés - celui du "bon sauvage» qu'il aurait été et dont il joue - rousseauiste : la guerre n'est pas l'état normal de l'homme, mais l'effet de son intoxication par les mensonges, la « tradition », en somme la mauvaise éducation et les mauvais maitres ${ }^{16}$.

Il se fait envoyer par ses sœurs tout ce qu'elles peuvent lui procurer de ce qui est publié. La liste de ses lectures que sa correspondance de guerre dévoile prouve sa curiosité et sa volonté de dénoncer le travestissement, même littéraire. C'est un devoir qu'il se fixe au nom de ses camarades, les simples soldats dont il se distingue, qui le heurtent souvent par leur attitude mais qu'il prend peu à peu en sympathie parce qu'il leur trouve plus de courage, de sincérité, d'humanité que chez les littérateurs ou les propagandistes qui participent au «bourrage de crâne »: "Un des vœux les plus ardents du poilu, souvent répétés dans les souvenirs de guerre, était qu'on sût un jour, après la guerre pensait-il, la vérité entière sur sa guerre ${ }^{17}$.» Il n'a de cesse dans ses lettres de dénoncer ce qui est « la chose la plus haïssable » (6 mai 1918) : « la littérature » qui cherche seulement à flatter le public $^{18}$. Pour lui, à la différence de son frère Loyalty, la guerre ne peut être considérée comme "matière à littérature ". Il ajoute dans cette même carte qu'il écrit à sa sœur Alice du 22 novembre 1917 : «Je considère comme sacrilège de faire avec notre sang et nos angoisses de la matière à littérature. Si nous avons encore la guerre au $\mathrm{XX}^{\mathrm{e}}$ siècle, c'est parce que les hommes ont trop entretenu cette fausse beauté du carnage ${ }^{19}{ }^{\prime}$. Dès le 6 juillet 1915 , dans une longue lettre écrite " aux tranchées », il avait décrit ce qu'il ressentait à l'égard « de la drogue concoctée dans les bureaux de rédaction »:

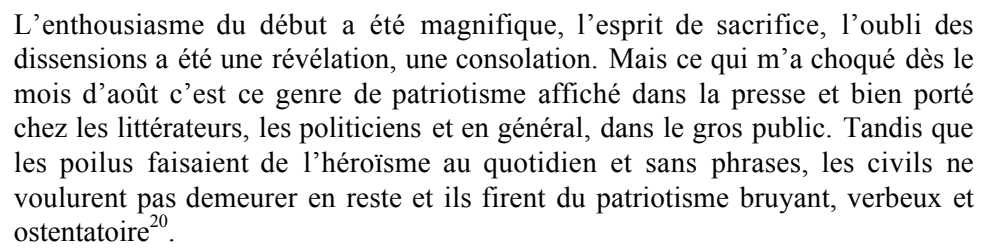
dissensions a été une révélation, une consolation. Mais ce qui m'a choqué dès le mois d'août c'est ce genre de patriotisme affiché dans la presse et bien porté chez les littérateurs, les politiciens et en général, dans le gros public. Tandis que les poilus faisaient de l'héroïsme au quotidien et sans phrases, les civils ne voulurent pas demeurer en reste et ils firent du patriotisme bruyant, verbeux et ostentatoire $^{20}$.

Bien entendu, sans varier dans ses convictions patriotiques, fier de se conduire en bon soldat et d'avoir pu conduire ses hommes au combat (4 juillet 1916) ${ }^{21}$, il aspire à la fin du massacre, ce qui est une attitude générale. Tout en participant à la guerre, comme la plupart, il en souhaite la fin. Son «pacifisme» - comme le pacifisme ancien combattant en général -

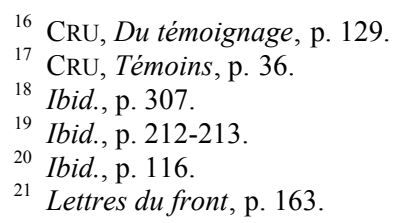


n'est pas une reconstruction de l'après-guerre. Il est né dans les tranchées et son œuvre qui veut servir la cause de la paix mûrit au même moment, même si elle n'est réalisée que dans les années qui suivent.

\section{Faussaires et "bons" témoins}

Témoigner pour Cru, c'est donc révéler la guerre vraie, dénoncer les mensonges qui la masquent, qui en embellissent la réalité, qui trompent le lecteur comme lui a été trompé par la littérature épique qui a nourri ses humanités. Témoins est un fort volume de plus de sept cents pages : les soixante-dix premières rassemblent l'introduction et l'exposition de la méthode, les six cents suivantes passent au crible trois cents ouvrages de guerre français édités à Paris et répartis en cinq catégories : journaux, souvenirs, réflexions, lettres et romans. Les dernières pages sont dévolues aux annexes, dont des tableaux classant œuvres et auteurs. Le plus significatif de ces tableaux est le premier, où Cru répartit les auteurs en six classes, de la moins fiable à la plus fiable. Ces auteurs ont été sélectionnés parmi ceux qui ont réellement combattu. Cru exclut les non-combattants et les officiers supérieurs qui étaient trop éloignés du front pour être des observateurs crédibles. Témoins n'entend rassembler que « les relations des narrateurs qui ont agi et vécu les faits, à l'exclusion des récits des spectateurs, qu'ils soient du quartier général à quelques kilomètres de la scène ou dans leur bureau à Paris $^{22} »$.

Du point de vue de la critique littéraire ou de l'analyse du témoignage telle qu'on la conçoit aujourd'hui, ses méthodes sont évidemment obsolètes. Sa critique est platement positiviste. Elle est bâtie à l'aune d'une vérité mesurable, celle de l'expérience de guerre des combattants. Il se veut censeur impitoyable et il est prompt à disqualifier les récits pour des raisons qui ne sont pas historiquement fondées. Le témoignage qu'il prend en considération est à l'image du poilu qu'il préfère, un poilu idéal, qui correspond à des critères de qualité et, même, d'éducation. Comme on l'a fréquemment souligné, Témoins est le « récit codé sur sa propre expérience ${ }^{23} »$. Il passe à côté de ce qui, pour nous, fait l'intérêt principal de la littérature, c'est-à-dire la façon de transformer les faits en représentation, ce qui ne peut que surprendre pour un professeur de littérature. Mais sa critique ne se veut pas littéraire. Comme on l'a vu, il ne cesse de dénoncer « la littérature » qui est pour lui déformation de la vérité : «Le roman de guerre par les littérateurs civils est un fléau de la vérité historique au même titre que les légendes que j'essaie de discréditer ${ }^{24}$. Il conçoit avant tout la question de la mise en scène

${ }^{22}$ CRU, Témoins, p. 9.

${ }^{23}$ Leonard V. SMITH, «Jean Norton Cru, lecteur de livres de guerre », Annales du Midi, t. $112, n^{\circ} 232$, octobre-décembre 2000, p. 527.

${ }^{24}$ Cru, Témoins, p. 50. 
des souvenirs sous l'angle de la déformation qu'il redoute et qu'il entend dénoncer, la recherche de l'effet littéraire ou l'exaltation héroïque.

De ce fait, le succès ou la notoriété d'un auteur ne lui donne aucun avantage particulier. Il n'est pas meilleur témoin à ses yeux qu'un autre témoin moins connu. Roland Dorgelès, l'auteur des Croix de bois (1919), Henri Barbusse, celui du Feu (1916), sont comme Georges Duhamel (notamment pour la Vie des Martyrs, 1917), Jean Giraudoux (Lecture pour une ombre, 1917), Pierre Mac Orlan (Les poissons morts, 1917), etc. dans la classe 4, Jean Paulhan (Le guerrier appliqué, 1917) en classe 5 et Montherlant (Le Songe 1922) dans la dernière. Barbusse l'exaspère en raison d' « une interprétation tendancieuse, malveillante, déformante des faits ». Il entretient " des idées légendaires si chères aux chauvins de l'arrière : massacre des Boches qu'on tire comme des lapins, qu'on égorge comme des moutons ; rôle prépondérant de la baïonnette et du couteau ; attaques allemandes faites au coude à coude, à plusieurs rangs serrés; corps à corps où des grappes d'hommes forment une mêlée », etc. "Toutes ces sottises et bien d'autres, Barbusse les répète après les matamores du passé ou de l'arrière ». En outre, « ses personnages, comme ceux de Zola, n'ont aucune psychologie, ce sont des fantoches ${ }^{25}$ ». Il lui reproche surtout d'être un idéologue, un pacifiste qui dessert la paix par ses outrances. Il lui fait ce reproche dès la première lecture du $\mathrm{Feu}$. Il écrit à son propos le 23 mai 1917 : «Livre très vendu, mais que je n'approuve pas. Ce que Th. Hardy a fait pour la société civile, Barbusse le fait pour les soldats. Il ne voit que le laid et le souligne 3 fois $^{26}$. » Il fait le même reproche à Erich Maria Remarque, auquel il consacre une courte note : «Roman pacifiste ayant tous les défauts du genre représenté par Barbusse et Latzko : outrance du macabre, meurtre à l'arme blanche, ignorance de ce que tout fantassin combattant doit $\operatorname{savoir}^{27} »$. La critique est encore plus rude pour Roland Dorgelès : «Barbusse se trompe par fanatisme, Dorgelès se trompe en sacrifiant tout à la littérature », Barbusse est sincère, « il écrit pour défendre sa foi. Qui oserait en dire autant de Dorgelès ? Quand il imite $L e F e u$, c'est le succès du livre qu'il veut surtout imiter ${ }^{28}$ ». S'il pourfend les "mauvais" pacifistes - non parce qu'ils sont pacifistes, mais parce qu'ils déforment la réalité de la guerre et ne servent donc pas la cause qu'ils prétendent défendre -, il va de soi qu'il n'a pas d'indulgence pour les bellicistes et qu'il démonte la "version miraculeuse et mystique » des événements ${ }^{29}$ - celle de Maurice Barrès - ou les divagations de Montherlant qu'il ne faut pas prendre

Ibid., p. 564.

${ }^{26}$ Lettres du front, p. 246.

${ }^{27}$ CRU, Témoins, p. 80. Andreas Latzko est l'auteur de Hommes en guerre (1917).

${ }^{28}$ CRU, Témoins, p. 588.

${ }^{29}$ Ibid., p. 378 à propos des récits de Jacques Péricard, auteur de l'apostrophe rendue célèbre par la propagande de «Debout les morts!», titre qu'il a donné récit qu'il publie en 1918. Cru considère que cette « légende » est « la raison d'être des livres de Péricard ». 
trop au sérieux. Dans une lutte individuelle son héros tue l'Allemand et la scène est décrite avec des détails sadiques (p. 124). Je suis loin de croire à la réalité de l'incident et des sensations : l'auteur croit être dans le ton de la mode ; ce qu'il écrit là, ce sont des mots qui font bien, pense-t-il, les mots que demande ce passage de bravoure. ${ }^{30}$

Il n'est pas plus indulgent pour Jean Paulhan qui a écrit : « Nous courions vers eux le fusil serré dans les mains et la haine haute... » (Le guerrier appliqué, 1917). Récusant la réalité de cette haine, Cru s'interroge : "Cette haine si fortement matérialisée ici, Paulhan l'a-t-il éprouvée ? N'est-ce pas plutôt un de ces mots qui font bien, qui rendent bien en littérature ${ }^{31}$ ? » Cru sait surmonter ses sentiments. Il le montre à propos de Clavel soldat de Léon Werth (1919), « livre laid, déplaisant, irritant même par l'impertinence de ses paradoxes », qui manque de goût, qui «abuse des termes crus », qui méprise les paysans que cet écrivain connaît peu, qui pêche par orgueil puisque Clavel ne connaît pas la peur. Mais ce livre qu'il serait tenté de mettre «bien en dessous de tous les autres romans de guerre « «est appuyé sur une expérience réelle que l'auteur n'a pas transposée ni modifiée dans un but littéraire ${ }^{32}$ ». C'est un roman vrai, vrai dans le sens où Cru l'entend. Mais l'auteur qu'il place au-dessus de tous les autres, c'est Maurice Genevoix. Sous Verdun l'enthousiasme dès 1916. Il écrit à sa sœur le 10 novembre 1916 que Genevoix donne "des idées exactes sur un sujet où je n'ai rien vu de satisfaisant à ce jour. Ce n'est ailleurs que littérature, paroles vaines, procédés, efforts pour viser l'effet et incapacité à voir. Voir, tout est là et ce jeune Normalien a $\underline{v u}^{33} »$. Il garde la même appréciation dans Témoins : «Parmi tous les auteurs de la guerre Genevoix occupe le premier rang, sans conteste », et, très significativement, il explique que « ce n'est pas le jugement d'un lecteur qui préfère un roman à un autre ", mais "plutôt le jugement qui accorde la mention très honorable à une seule d'entre plusieurs thèses ». Genevoix

a su raconter sa campagne de huit mois avec la plus scrupuleuse exactitude en s'interdisant tout enjolivement dû à l'imagination, mais cependant en ressuscitant la vie des événements et des personnes, des âmes et des opinions, des gestes et des attitudes, des paroles et des conversations. Son récit est l'image fidèle d'une vie qui fut vécue, comme un bon roman est l'image d'une vie fictive mais vraisemblable. Aucun récit de guerre ne ressemble plus à un roman... ${ }^{34}$

${ }^{30}$ Ibid., p. 632 à propos du roman Le songe (1922).

${ }^{31}$ Ibid., p. 638.

32 Ibid., p. 653.

${ }^{33}$ Lettres du front, p. 194.

${ }^{34}$ CRU, Témoins, p. 145. 


\section{Jean Norton Cru historien}

En dépit de ses a priori, de ses maniaqueries et de ses œillères, l'œuvre de Jean Norton Cru ne mérite pas l'opprobre à laquelle on la voue parfois. Ses jugements ne manquent pas tous de finesse. Sa curiosité est réelle. Son souci de dire d'où il parle, qui il est et de faire de même pour «ses » témoins, qu'il situe sur le front avec le maximum de précisions, nous paraît tout à fait remarquable.

S'il témoigne à travers le témoignage des autres, c'est pour fonder la vérité combattante sur la guerre d'une façon qu'il croit irréfutable, ce qui ne serait pas le cas, à ses yeux, s'il écrivait à la première personne. Son ambition est de se situer au-dessus de la mêlée des témoins ordinaires, "faire un faisceau des témoignages des combattants sur la guerre ${ }^{35} »$, faire œuvre de science. Témoins, c'est pour lui le témoignage absolu car reposant sur cette science du témoignage qu'il entend proposer à la communauté universitaire. Il ne récuse pas la subjectivité des récits, mais veut dégager une vérité commune, objective, tirée de la répétition d'éléments - faits, attitudes, sentiments - présents dans l'expérience de ses camarades - les "vrais" combattants - et que la sienne confirme.

Avec l'ambition humaine, éthique et scientifique qui est la sienne, Cru veut être le passeur qui donnera les clés de la compréhension à ceux qui n'ont pas vécu la guerre et qui tenteront pourtant d'en rendre compte. Il entend servir l'histoire et lui donner le matériau "propre », épuré de ses scories, et les outils qui lui permettront de trier le vrai et le faux. Sa démarche oscille, avec ses limites évidentes, entre une posture d'historien, aller au-delà des mythes, retrouver la réalité de la guerre par-delà les habillages rhétoriques et la propagande, et la position, dominante chez lui, du témoin, sûr de sa vérité et doutant que ceux qui n'ont pas vécu les événements soient capables de les comprendre : " on ne saurait attendre de l'historien qu'il débrouille ce chaos $^{36} »$. Du coup, puisque l'on se méfie a priori de la capacité de ceux qui écriront l'histoire, il faut assurer soi-même la transmission de la vérité. Toute la problématique du "devoir de mémoire » dont le mouvement ancien combattant se fait aujourd'hui encore une spécialité est contenue là.

Témoins marque une date dans l'historiographie puisque l'ouvrage contribue à inscrire le témoignage en dignité comme une source à prendre en compte avec sérieux, alors que l'histoire "noble", celle des universitaires, ne tenait pas encore cela pour acquis. Elle en fait le révélateur d'une vérité humaine que l'histoire officielle, l'histoire militaire en particulier, néglige.

Cette œuvre est aussi le reflet des sentiments et des idées que nombre de combattants ont adoptés au cours de la lutte, qu'ils ont extériorisés après, et qu'ils expriment dans un rejet viscéral de la guerre, comme Antoine Prost l'a

\footnotetext{
${ }^{35}$ Ibid., p. 13.

${ }^{36}$ Ibid., p. 21.
} 
fait comprendre voilà trente ans ${ }^{37}$. Cru participe si pleinement de cette évolution et de ce positionnement qu'il reproduit le clivage - classique entre ceux qui se considèrent comme les « vrais » combattants, dont il estime faire partie, qui, seuls, peuvent témoigner (au sens le plus fort du mot), et les autres, qui, a priori sont disqualifiés pour dire la guerre réelle. Son œuvre est d'abord un témoignage, particulier, sur une représentation devenue dominante - et sans doute très tôt - parmi les rescapés du conflit. L'accueil qu'il reçoit parmi les anciens combattants s'explique par cette communauté de sentiments qui lui fait écrire en 1924: «L'immense majorité d'entre nous n'avait pas voulu la guerre et en la faisant nous ne sommes devenus ni brutes ni esclaves $^{38}$. » Cette œuvre est par anticipation la réfutation de la thèse sur la brutalisation qui aurait marqué de façon indélébile les combattants et la société et dont Antoine Prost, au moins pour la France, a montré les limites ${ }^{39}$.

\footnotetext{
${ }^{37}$ Dans sa thèse : Antoine Prost, Les Anciens combattants et la société française 19141939, Paris, Presses de la FNSP, 1977.

${ }_{38}^{38}$ Cité par sa sœur: VOGEL, Annales de la Faculté, p. 63.

39 Cette thèse est issue des travaux de George L. Mosse, notamment Fallen soldiers: Reshaping the Memory of the World War, édité en français sous le titre De la Grande Guerre au totalitarisme. La brutalisation des sociétés européennes, Paris, Hachette, 1999. Voir Antoine PROST, «Les limites de la brutalisation. Tuer sur le front occidental, 1914-1918 », Vingtième siècle, Revue d'histoire, 2004/1, nº 81, p. 5-20.
} 